\title{
Increasing Mean Inactivity Time Ordering: A Quantile Approach
}

\author{
M. Kayid $(D),{ }^{1,2}$ S. Izadkhah $(D),{ }^{3}$ and A. Alfifi ${ }^{1}$ \\ ${ }^{1}$ Department of Statistics and Operations Research, College of Science, King Saud University, P.O. Box 2455, Riyadh 11451, Saudi Arabia \\ ${ }^{2}$ Department of Mathematics and Computer Science, Faculty of Science, Suez University, Suez 41522, Egypt \\ ${ }^{3}$ Department of Statistics, Faculty of Basic Science and Engineering of Bijar, University of Kurdistan, Bijar, Iran
}

Correspondence should be addressed to M. Kayid; el_kayid2000@yahoo.com

Received 18 September 2017; Revised 3 March 2018; Accepted 25 March 2018; Published 26 April 2018

Academic Editor: Alessandro Lo Schiavo

Copyright (C) 2018 M. Kayid et al. This is an open access article distributed under the Creative Commons Attribution License, which permits unrestricted use, distribution, and reproduction in any medium, provided the original work is properly cited.

\begin{abstract}
We study further the quantile mean inactivity time order. Relations between the proposed stochastic order and the other transform stochastic orders are obtained. Besides, sufficient conditions for the stochastic order are provided. Then, preservation of the order under monotone transformations, series, and parallel systems and mixtures of a general family of semiparametric distributions is studied. Examples are also given to illustrate the results.
\end{abstract}

\section{Introduction}

Comparisons of random variables according to stochastic orders have played a central role in reliability theory, risk theory, and other fields. There are many stochastic orders proposed in the past years giving rise to a large body of literature (cf. Shaked and Shanthikumar [1], Müller and Stoyan [2], and Belzunce et al. [3]). In order to compare the aging properties of two arbitrary life distributions, several stochastic orders, known as transform orders, providing new relationships among several popular aging notions, have been introduced (see, e.g., Nair et al. [4] and Nanda et al. (2016) and the references therein). Consider two continuous random variables $X$ and $Y$ with distribution functions $F$ and $G$ and quantile functions $F^{-1}(p)=\inf \{x \mid F(x) \geq p\}$ and $G^{-1}(p)=$ $\inf \{x \mid G(x) \geq p\}$, respectively, for any value $p \in(0,1)$. Denote by $\operatorname{supp}(X)$ and $\operatorname{supp}(Y)$ the support of the random variables $X$ and $Y$, respectively, which are assumed to be intervals. One of the strongest transform stochastic orders is the convex transform order. Van Zwet [5] proposed a skewness order, called the convex transform order, which captures the property of one distribution being more skewed than the other. It is said, according to their work, that $X$ is smaller than $Y$ of the convex transform order (denoted by $X \leq_{c} Y$ ) when

$$
G^{-1}(F(x)) \text { is convex in } x \in \operatorname{supp}(X) \text {. }
$$

For more properties of the convex transform order in reliability and actuarial studies we refer the readers to Barlow and Proschan (1981), Marshall and Olkin [6], Shaked and Shanthikumar [1], Kochar and $\mathrm{Xu}$ [7], and Barmalzan and Payandeh Najafabadi [8] among others. In terms of aging notions of lifetime distributions (that have 0 as the common left endpoint of their supports) Kochar and Wiens [9] called the order " $\leq_{c}$ " the more increasing failure rate (IFR) order which is equivalent to

$$
\frac{r_{X}\left(F^{-1}(p)\right)}{r_{Y}\left(G^{-1}(p)\right)} \text { is increasing in } p \in(0,1),
$$

where

$$
\begin{aligned}
& r_{X}(t)=\frac{f(t)}{\bar{F}(t)}, \text { for } t: \bar{F}(t)>0, \\
& r_{Y}(t)=\frac{g(t)}{\bar{G}(t)}, \quad \text { for } t: \bar{G}(t)>0
\end{aligned}
$$

are the failure rates of $X$ and $Y$, respectively, provided that $F$ and $G$ are absolutely continuous with associated density functions $f$ and $g$ and survival functions $\bar{F}=1-F$ and $\bar{G}=$ $1-G$. In the literature, several weaker transform orders have also been proposed to compare the relative aging properties. Kochar and Wiens [9] proposed another stochastic order, for 
describing the aging phenomenon, called decreasing mean residual life order. We say $X$ is smaller than $Y$ of the decreasing mean residual life order (denoted by $X \leq_{\mathrm{dmrl}} Y$ ) whenever

$$
\frac{\mu_{Y}\left(G^{-1}(p)\right)}{\mu_{X}\left(F^{-1}(p)\right)} \text { is increasing in } p \in(0,1),
$$

in which

$$
\begin{aligned}
& \mu_{X}(t)=E(X-t \mid X>t)=\frac{\int_{t}^{\infty} \bar{F}(x) d x}{\bar{F}(t)} \\
& \text { for } t: \bar{F}(t)>0, \\
& \mu_{Y}(t)=E(Y-t \mid Y>t)=\frac{\int_{t}^{\infty} \bar{G}(x) d x}{\bar{G}(t)} \\
& \text { for } t: \bar{G}(t)>0
\end{aligned}
$$

are respective mean residual life (MRL) functions of $X$ and $Y$ (cf. Lai and Xie [10] for reliability properties of the MRL functions). Kochar and Wiens [9] showed that if $\operatorname{supp}(X)=\operatorname{supp}(Y)=[0, a)$, where $a \in(0, \infty)$, then

$$
\begin{aligned}
& X \leq_{c} Y \Longrightarrow \\
& X \leq_{\mathrm{dmrl}} Y .
\end{aligned}
$$

For further properties of the order " $\leq_{\mathrm{dmrl}}$ " we refer the readers to Kochar and Wiens [9], Kochar [11], Shaked and Shanthikumar [1], and Kang and Yan [12]. Another weaker stochastic order is the star order. We say $X$ is smaller than $Y$ of the star order (denoted by $X \leq_{*} Y$ ) whenever

$$
\frac{G^{-1}(p)}{F^{-1}(p)} \text { is increasing in } p \in(0,1) \text {. }
$$

From (4.B.3) in Shaked and Shanthikumar [1],

$$
\begin{aligned}
& X \leq_{c} Y \Longrightarrow \\
& X \leq_{*} Y .
\end{aligned}
$$

One can see Bartoszewicz [13], Li and Xu (2004), Boland et al. [14], Bartoszewicz and Skolimowska [15], Bartoszewicz and Skolimowska [16], and Kochar and $\mathrm{Xu}$ [17] to find further properties of the star order in the context of reliability theory. In the context of transform orders, Belzunce et al. [18] introduced a new criterion to compare risks based on the notion of expected proportional shortfall which is useful for comparing risks of different nature free of the base currency. The aim of the current investigation is to develop the study of another transform order closely related to the convex transform and the star orders, proposed by Arriaza et al. [19]. This stochastic order is similar to the order " $\leq_{\mathrm{dmrl}}$ " but considers mean inactivity times at quantiles instead of the quantile mean residual lives of the units.

\section{Main Results}

In this section, we have brought our main achievements. We first recall the stochastic order and its relationships with some other well-known stochastic orders. Then preservation of the order under monotone transformations, series systems, parallel systems, and mixtures of a typical family of semiparametric distributions is investigated in detail. Some examples are also included to enhance the study of the results of this section. For a nonnegative random variable $X$ with distribution function $F$, the mean inactivity time (MIT) of $X$ is defined as (cf. Kayid and Ahmad [20])

$$
\phi_{X}(t)=E[t-X \mid X \leq t]=\frac{\int_{0}^{t} F(x) d x}{F(t)},
$$

$$
t: F(t)>0 \text {, }
$$

and similarly the MIT of $Y$ having distribution $G$ is given by

$$
\begin{aligned}
\phi_{Y}(t)=E[t-Y \mid Y \leq t]=\frac{\int_{0}^{t} G(x) d x}{G(t)}, & \\
& t: G(t)>0 .
\end{aligned}
$$

To relate the MIT of two lifetime units with their ages, the MITs could be evaluated at the quantiles of the underlying distributions. Given that the failure of the unit A has occurred before or at a time point $t$, at which $F(t)=p$ and the failure of unit B has taken place before or at a time point $t$, at which $G(t)=p$, the MIT functions of random lifetime $X$ of the unit $\mathrm{A}$ and random lifetime $Y$ of the unit $\mathrm{B}$ are reduced to

$$
\begin{aligned}
& \phi_{X}\left(F^{-1}(p)\right)=\frac{1}{p} \int_{0}^{F^{-1}(p)} F(x) d x, \\
& \phi_{Y}\left(G^{-1}(p)\right)=\frac{1}{p} \int_{0}^{G^{-1}(p)} G(x) d x,
\end{aligned}
$$

respectively. According to Nair et al. [4], for each $p \in(0,1)$, $M_{X}(p)=\phi_{X}\left(F^{-1}(p)\right)$ and $M_{Y}(p)=\phi_{Y}\left(G^{-1}(p)\right)$ are called quantile MITs of $X$ and $Y$. There is a stochastic order in the literature called location-independent riskier order that has been introduced by Jewitt [21] to compare random assets in risk analysis, which is equivalent to comparison of quantile MIT functions. Conventionally, $X$ is said to be less than $Y$ in the location-independent riskier order (denoted by $X \leq_{\text {lir }} Y$ ) if

$$
\int_{0}^{F^{-1}(p)} F(x) d x \leq \int_{0}^{G^{-1}(p)} G(x) d x, \quad \forall p \in(0,1) .
$$

It is trivial to see that this is equivalent to having $M_{X}(p) \leq$ $M_{Y}(p)$, for all $p \in(0,1)$. We are now ready to establish the comparison of lifetime random variables according to the ratio of their mean inactivity times at quantiles and then present our main results about the stochastic order. To be in agreement with the name of the dual order, that is, the decreasing mean residual life order, we call the quantile mean inactivity time order introduced by Arriaza et al. [19] the increasing mean inactivity time order. We bring some useful lemmas that will be used throughout this section. 
Definition 1. Suppose $X$ and $Y$ are two nonnegative random variables having mean inactivity time functions $\phi_{X}$ and $\phi_{Y}$, respectively. It is said that quantile mean inactivity time of $Y$ is more increasing than quantile mean inactivity time of $X$ (written as $X \leq_{\text {imit }} Y$ ) whenever

$$
\frac{\phi_{Y}\left(G^{-1}(p)\right)}{\phi_{X}\left(F^{-1}(p)\right)} \text { is increasing in } p \in(0,1),
$$

or equivalently if

$$
\frac{\int_{0}^{G^{-1}(p)} G(x) d x}{\int_{0}^{F^{-1}(p)} F(x) d x} \text { is increasing in } p \in(0,1) .
$$
results.

The following lemmas will be useful to derive some of our

Lemma 2 (Barlow and Proschan (1975, p. 120)). Let $W$ be a measure on the interval $(\alpha, \beta)$, not necessarily nonnegative. Let $h$ be a nonnegative function defined on $(\alpha, \beta)$.

(i) If $\int_{t}^{\beta} d W(x) \geq 0$, for all $t \in(\alpha, \beta)$, and ifh is increasing, then $\int_{\alpha}^{\beta} h(x) d W(x) \geq 0$.

(ii) If $\int_{\alpha}^{t} d W(x) \geq 0$, for all $t \in(\alpha, \beta)$, and if $h$ is decreasing, then $\int_{\alpha}^{\beta} h(x) d W(x) \geq 0$.

Lemma 3 (basic composition formula, Karlin [22, p. 17]). Let $g$ be a TP $P_{2}$ function in $(x, z) \in \chi \times \zeta$ and let $h$ be a $T P_{2}$ function in $(z, y) \in \zeta \times \gamma$. Then the function $f$ given by

$$
f(x, y)=\int_{\zeta} g(x, z) h(z, y) d \sigma(z)
$$

is $T P_{2}$ in $(x, y) \in \chi \times \gamma$, where $\chi, \zeta$, and $\gamma$ are real subsets of $R$ and $\sigma$ is a $\sigma$-finite measure.

2.1. Relation with Other Stochastic Orders. Let $X$ and $Y$ be two random variables with respective absolutely continuous distribution functions $F_{1}$ and $F_{2}$ which are assumed to be strictly increasing. Then

$$
\begin{gathered}
X \leq_{\text {imit }} Y \Longleftrightarrow \\
\int_{0}^{p} q d F_{i}^{-1}(q) \text { is } \mathrm{TP}_{2} \text { in }(i, p),
\end{gathered}
$$

when $i=1,2$ and $p \in(0,1)$. Let $X_{i}$ have density function $f_{i}$, $i=1,2$. Then it is possible to verify that

$$
\begin{aligned}
\int_{0}^{p} q d F_{i}^{-1}(q) & =\int_{0}^{p} \frac{q}{f_{i}\left(F_{i}^{-1}(q)\right)} d q \\
& =\int_{0}^{1} \frac{q}{f_{i}\left(F_{i}^{-1}(q)\right)} I[q<p] d q .
\end{aligned}
$$

Now, one can see that $X \leq_{c} Y$ yields $q / f_{i}\left(F_{i}^{-1}(q)\right)$ which is $\mathrm{TP}_{2}$ in $(i, q) \in\{1,2\} \times(0,1)$ and also it is evident that $I[q<p]$ is
$\mathrm{TP}_{2}$ in $(q, p) \in(0,1) \times(0,1)$. Thus, an application of Lemma 3 leads to $X \leq_{\text {imit }} Y$. For the case where $X$ and $Y$ do not have absolutely continuous distributions we demonstrate the same result as follows.

Theorem 4 (Arriaza et al. [19]). Let $X$ and $Y$ be two continuous nonnegative random variables. Then

$$
\begin{aligned}
& X \leq_{c} Y \Longrightarrow \\
& X \leq_{\text {imit }} Y .
\end{aligned}
$$

From (4.B.5) in Shaked and Shanthikumar [1], $X \leq_{c} Y$ if, and only if, $f\left(F^{-1}(p)\right) / g\left(G^{-1}(p)\right)$ increases in $p \in(0,1)$. Thus, as stated in Theorem 4 this is a sufficient condition for the increasing mean inactivity time order. In the following result we provide some other sufficient conditions for the order " $\leq_{\text {imit }}$ " such that the order " $\leq_{c}$ " does not hold.

Theorem 5. Let $X$ and $Y$ be two absolutely continuous nonnegative random variables having interval supports and finite means which have strictly increasing distribution functions. If

(i) there exists $p_{0} \in(0,1)$ such that $f\left(F^{-1}(p)\right) / g\left(G^{-1}(p)\right)$ increases in $p<p_{0}$,

(ii) $f\left(F^{-1}(p)\right) / g\left(G^{-1}(p)\right)$ strictly decreases in $p \geq p_{0}$,

(iii) $\left(E(Y)-G^{-1}(p)\right) /\left(E(X)-F^{-1}(p)\right)$ increases in $p \epsilon$ $\left[p_{0}, 1\right)$, then

$$
X \leq_{\text {imit }} Y
$$

but $X \Varangle_{c} Y$.

Proof. First, we consider two arbitrary values $q$ and $p$ such that $0 \leq q<p<p_{0}$. The assumption given in (i) implies that

$$
\frac{q d G^{-1}(q)}{f\left(F^{-1}(p)\right)} \leq \frac{q d F^{-1}(q)}{g\left(G^{-1}(p)\right)},
$$

and, therefore,

$$
\int_{0}^{p} \frac{q d F^{-1}(q)}{g\left(G^{-1}(p)\right)} \geq \int_{0}^{p} \frac{q d G^{-1}(q)}{f\left(F^{-1}(p)\right)}, \quad \forall p \in\left(0, p_{0}\right) .
$$

Now, consider $p<q \in\left[p_{0}, 1\right)$. Assumption (ii) provides that

$$
\frac{f\left(F^{-1}(p)\right)}{g\left(G^{-1}(p)\right)} \geq \frac{(1-q) f\left(F^{-1}(q)\right)}{(1-q) g\left(G^{-1}(q)\right)},
$$

and further that

$$
\begin{aligned}
& \frac{f\left(F^{-1}(p)\right)}{g\left(G^{-1}(p)\right)} \geq \frac{\int_{p}^{1}(1-q) d G^{-1}(q)}{\int_{p}^{1}(1-q) d F^{-1}(q)} \\
& =\frac{\int_{0}^{1}(1-q) d G^{-1}(q)-\int_{0}^{p} q(1-q) d G^{-1}(q)}{\int_{0}^{1}(1-q) d F^{-1}(q)-\int_{0}^{p} q(1-q) d F^{-1}(q)} \\
& =\frac{E(Y)-G^{-1}(p)+\int_{0}^{p} q d G^{-1}(q)}{E(X)-F^{-1}(p)+\int_{0}^{p} q d F^{-1}(q)}, \quad \forall p \geq p_{0},
\end{aligned}
$$


which holds if, and only if,

$$
\begin{aligned}
& \int_{0}^{p} \frac{q d F^{-1}(q)}{g\left(G^{-1}(p)\right)}-\int_{0}^{p} \frac{q d G^{-1}(q)}{f\left(F^{-1}(p)\right)} \\
& \quad \geq \frac{E(Y)-G^{-1}(p)}{f\left(F^{-1}(p)\right)}-\frac{E(X)-F^{-1}(p)}{g\left(G^{-1}(p)\right)} \\
& \quad=\left(E(X)-F^{-1}(p)\right)^{2} \frac{\partial}{\partial p} \frac{E(Y)-G^{-1}(p)}{E(X)-F^{-1}(p)}, \\
& \quad p \in\left[p_{0}, 1\right),
\end{aligned}
$$

which is nonnegative from (iii). That is, we proved that

$$
\int_{0}^{p} \frac{q d F^{-1}(q)}{g\left(G^{-1}(p)\right)} \geq \int_{0}^{p} \frac{q d G^{-1}(q)}{f\left(F^{-1}(p)\right)}, \quad \forall p \in(0,1)
$$

which means that $X \leq_{\text {imit }} Y$. Assertions (i) and (ii) ensure that $f\left(F^{-1}(p)\right) / g\left(G^{-1}(p)\right)$ is not increasing in $p$, for all values $p$ in $(0,1)$. Hence, $X \Varangle_{c} Y$ and the proof is obtained.

The sufficient conditions of Theorem 5 are in the spirit of some previous results established by Belzunce et al. [23] and Belzunce and Martínez-Riquelme [24]. The next example applies Theorem 5 .

Example 6. Consider two nonnegative random variables $X$ and $Y$ with respective quantile functions $F^{-1}(p)=p^{0.5}$ and $G^{-1}(p)=1-(1-p)^{1.01}$ for $p \in[0,1]$. Since $f\left(F^{-1}(p)\right) / g\left(G^{-1}(p)\right)=2.02 p^{0.5}(1-p)^{0.01}$, thus there exists the point $p_{0}=50 / 51$ such that $f\left(F^{-1}(p)\right) / g\left(G^{-1}(p)\right)$ is increasing in $p \in\left(0, p_{0}\right)$ and it is strictly decreasing in $p \in$ $\left[p_{0}, 1\right)$. That is, conditions (i) and (ii) in Theorem 5 hold true. It is also possible to see that $E(X)=2 / 3$ and $E(Y)=100 / 201$ and that $\left((1-p)^{1.01}-100 / 201\right) /\left(2 / 3-p^{0.5}\right)$ is increasing on $p \in(50 / 51,1)$. Assumption (iii) in Theorem 5 is therefore satisfied. Hence, $X \leq_{\text {imit }} Y$ and $X \not_{c} Y$.

Theorem 7. Let $X$ and $Y$ be two nonnegative random variables with continuous distribution functions $F$ and $G$, respectively.

(i) If $F$ and $G$ are absolutely continuously associated with density functions $f$ and $g$, respectively, and if $\lim _{p \rightarrow 0^{+}}\left(f\left(F^{-1}(p)\right) / g\left(G^{-1}(p)\right)\right) \geq 1$, then

$$
\begin{aligned}
& X \leq_{\text {imit }} Y \Longrightarrow \\
& X \leq_{\text {lir }} Y .
\end{aligned}
$$

(ii) If $X$ and $Y$ have finite means such that $\lim _{p \rightarrow 1^{-}}\left(\left(G^{-1}(p)-E(Y)\right) /\left(F^{-1}(p)-E(X)\right)\right) \geq 1$, then

$$
\begin{aligned}
& X \leq_{\text {imit }} Y \Longrightarrow \\
& X \geq_{\text {lir }} Y .
\end{aligned}
$$

Proof. (i) From (14), $X \leq_{\text {imit }} Y$ implies that

$$
\begin{aligned}
\frac{\int_{0}^{G^{-1}(p)} G(x) d x}{\int_{0}^{F^{-1}(p)} F(x) d x} & \geq \lim _{p \rightarrow 0^{+}} \frac{\int_{0}^{G^{-1}(p)} G(x) d x}{\int_{0}^{F^{-1}(p)} F(x) d x} \\
& =\lim _{p \rightarrow 0^{+}} \frac{p / g\left(G^{-1}(p)\right)}{p / f\left(F^{-1}(p)\right)} \\
& =\lim _{p \rightarrow 0^{+}} \frac{f\left(F^{-1}(p)\right)}{g\left(G^{-1}(p)\right)}, \quad \forall p \in(0,1),
\end{aligned}
$$

which by assumption yields $\int_{0}^{G^{-1}(p)} G(x) d x \geq \int_{0}^{F^{-1}(p)} F(x) d x$, for all $p \in(0,1)$. That is, $X \leq_{\operatorname{lir}} Y$. (ii) Again, by using (14) we can conclude

$$
\begin{aligned}
\frac{\int_{0}^{G^{-1}(p)} G(x) d x}{\int_{0}^{F^{-1}(p)} F(x) d x} & \leq \lim _{p \rightarrow 1^{-}} \frac{\int_{0}^{p}\left(G^{-1}(p)-G^{-1}(q)\right) d q}{\int_{0}^{p}\left(F^{-1}(p)-F^{-1}(q)\right) d q} \\
& =\lim _{p \rightarrow 1^{-}} \frac{G^{-1}(p)-E(Y)}{F^{-1}(p)-E(X)},
\end{aligned}
$$

for $p \in(0,1)$,

together with assumption giving $\int_{0}^{G^{-1}(p)} G(x) d x \leq$ $\int_{0}^{F^{-1}(p)} F(x) d x$, for all $p \in(0,1)$; that is, $X \geq_{\text {lir }} Y$.

Corollary 8. Let $X$ and $Y$ be two nonnegative continuous random variables with finite support. If $u_{Y}-E(Y) \leq u_{X}-E(X)$ then $X \leq_{\text {imit }} Y$ implies $X \geq_{\text {lir }} Y$.

Theorem 9 (Arriaza et al. [19]). Let $X$ and $Y$ be two nonnegative random variables with absolutely continuous distributions. Then

$$
\begin{aligned}
& X \leq_{\text {imit }} Y \Longrightarrow \\
& X \leq_{*} Y .
\end{aligned}
$$

Remark 10. For any $p, q \in(0,1)$, denote $d W(q, p)=I[q<$ $p] w(q, p)$, where

$$
w(q, p)=q\left(\frac{d F^{-1}(q)}{g\left(G^{-1}(p)\right)}-\frac{d G^{-1}(q)}{f\left(F^{-1}(p)\right)}\right) .
$$

Now, $X \leq_{\text {imit }} Y$ implies that

$$
\int_{0}^{q^{*}} d W(q, p) \geq 0, \quad \forall p, q^{*} \in(0,1) .
$$

2.2. Monotone Transformations. Preservation of the increasing mean inactivity time order under increasing concave transformations is obtained as follows.

Theorem 11. Let $X$ and $Y$ be two nonnegative random variables with absolutely continuous distributions. If 
(i) $\phi$ is a nonnegative differentiable, strictly increasing, and concave function,

(ii) $\phi^{\prime}\left(G^{-1}(p)\right) / \phi^{\prime}\left(F^{-1}(p)\right)$ is increasing in $p \in(0,1)$, then

$$
\begin{gathered}
X \leq_{\text {imit }} Y \Longrightarrow \\
\phi(X) \leq_{\text {imit }} \phi(Y) .
\end{gathered}
$$

Proof. First, denote by $F_{\phi}, G_{\phi}, f_{\phi}$, and $g_{\phi}$ the distribution and density functions of $\phi(X)$ and $\phi(Y)$, respectively, which are given by

$$
\begin{aligned}
& F_{\phi}(t)=F\left(\phi^{-1}(t)\right), \\
& G_{\phi}(t)=G\left(\phi^{-1}(t)\right), \\
& t \geq 0, \\
& f_{\phi}(t)=\frac{f\left(\phi^{-1}(t)\right)}{\phi^{\prime}\left(\phi^{-1}(t)\right)}, \\
& g_{\phi}(t)=\frac{g\left(\phi^{-1}(t)\right)}{\phi^{\prime}\left(\phi^{-1}(t)\right)}, \\
& t \geq 0 .
\end{aligned}
$$

Therefore, for any $u \in(0,1)$, we have

$$
\begin{aligned}
& f_{\phi}\left(F_{\phi}^{-1}(u)\right)=\frac{f\left(F^{-1}(u)\right)}{\phi^{\prime}\left(F^{-1}(u)\right)}, \\
& g_{\phi}\left(G_{\phi}^{-1}(u)\right)=\frac{g\left(G^{-1}(u)\right)}{\phi^{\prime}\left(G^{-1}(u)\right)} .
\end{aligned}
$$

$\phi(X) \leq_{\text {imit }} \phi(Y)$ holds if, and only if,

$$
\int_{0}^{p} \frac{q d F_{\phi}^{-1}(q)}{g_{\phi}\left(G_{\phi}^{-1}(p)\right)} \geq \int_{0}^{p} \frac{q d G_{\phi}^{-1}(q)}{f_{\phi}\left(F_{\phi}^{-1}(p)\right)}, \quad \forall p \in(0,1),
$$

which holds if, and only if,

$$
\begin{aligned}
\int_{0}^{p} \frac{q \phi^{\prime}\left(G^{-1}(p)\right) \phi^{\prime}\left(F^{-1}(q)\right)}{g\left(G^{-1}(p)\right)} d F^{-1}(q) & \\
\geq \int_{0}^{p} \frac{q \phi^{\prime}\left(G^{-1}(q)\right) \phi^{\prime}\left(F^{-1}(p)\right)}{f\left(F^{-1}(p)\right)} d G^{-1}(q), & \\
& \forall p \in(0,1) .
\end{aligned}
$$

From (ii), for all $p>q$, the following holds:

$$
\begin{aligned}
& \phi^{\prime}\left(G^{-1}(p)\right) \phi^{\prime}\left(F^{-1}(q)\right) \\
& \quad \geq \phi^{\prime}\left(G^{-1}(q)\right) \phi^{\prime}\left(F^{-1}(p)\right) .
\end{aligned}
$$

Therefore, it suffices to prove that

$$
\begin{aligned}
& \int_{0}^{p} \frac{q \phi^{\prime}\left(G^{-1}(q)\right)}{g\left(G^{-1}(p)\right)} d F^{-1}(q) \\
& \quad \geq \int_{0}^{p} \frac{q \phi^{\prime}\left(G^{-1}(q)\right)}{f\left(F^{-1}(p)\right)} d G^{-1}(q), \quad \forall p \in(0,1) .
\end{aligned}
$$

For two arbitrary values $p$ and $q$ in $(0,1)$, consider the measure $W(q, p)$ is defined as in the proof of Theorem 9. From (32), the assumption that $X \leq_{\text {imit }} Y$ implies $\int_{0}^{q^{*}} d W(q, p) \geq 0$, for all $p, q^{*} \in(0,1)$. As is implied by assumption (ii), $\phi^{\prime}\left(G^{-1}(q)\right)$ is nonnegative and decreasing in $q \in(0,1)$. Hence Lemma 2 (ii) immediately gives

$$
\int_{0}^{1} \phi^{\prime}\left(G^{-1}(q)\right) d W(q, p) \geq 0, \quad \forall p, q \in(0,1),
$$

which makes (39) a valid statement. This ends the proof.

2.3. Series and Parallel Systems. We consider another reliability application of the imit order. Suppose that $P_{1}$ and $P_{2}$ denote two parallel systems and that $S_{1}$ and $S_{2}$ denote two series systems each consisting of $n$ i.i.d. components. Further, assume that $X_{1}, X_{2}, \ldots, X_{n}$ are i.i.d. lifetime random variables with distribution $F$ and that $Y_{1}, Y_{2}, \ldots, Y_{n}$ are i.i.d. lifetime random variables with distribution $G$. Denote by

$$
\begin{aligned}
& X_{n: n}=\max \left\{X_{1}, X_{2}, \ldots, X_{n}\right\}, \\
& Y_{n: n}=\max \left\{Y_{1}, Y_{2}, \ldots, Y_{n}\right\}
\end{aligned}
$$

the lifetimes of $P_{1}$ and $P_{2}$, respectively. Further,

$$
\begin{aligned}
& X_{1: n}=\min \left\{X_{1}, X_{2}, \ldots, X_{n}\right\}, \\
& Y_{1: n}=\min \left\{Y_{1}, Y_{2}, \ldots, Y_{n}\right\}
\end{aligned}
$$

denote the lifetimes of $S_{1}$ and $S_{2}$, respectively. Next we focus on relations between the imit ordering of two systems lifetimes and the imit ordering of their components lifetimes.

Theorem 12. Let $X$ and $Y$ be two nonnegative random variables with absolutely continuous distribution functions. Let $X_{1}, X_{2}, \ldots, X_{n}$ and $Y_{1}, Y_{2}, \ldots, Y_{n}$ be i.i.d. copies of $X$ and $Y$, respectively. Then

$$
\begin{gathered}
X \leq_{\text {imit }} Y \Longrightarrow \\
X_{1: n} \leq_{\text {imit }} Y_{1: n} .
\end{gathered}
$$

Proof. First, denote by $F_{1: n}$ and $G_{1: n}$ the distribution functions of $X_{1: n}$ and $Y_{1: n}$, respectively, given by

$$
\begin{aligned}
& F_{1: n}(t)=1-(1-F(x))^{n}, \\
& G_{1: n}(t)=1-(1-G(t))^{n}, \\
& t \geq 0,
\end{aligned}
$$

from which we get

$$
F_{1: n}^{-1}(u)=F^{-1}\left(1-(1-u)^{1 / n}\right), \quad u \in(0,1),
$$

and, similarly,

$$
G_{1: n}^{-1}(u)=G^{-1}\left(1-(1-u)^{1 / n}\right), \quad u \in(0,1)
$$


Therefore, if we denote by $f_{1: n}$ and $g_{1: n}$ the density functions of $X_{1: n}$ and $Y_{1: n}$, respectively, then for any $u \in(0,1)$ we have

$$
\begin{aligned}
& \frac{1}{f_{1: n}\left(F_{1: n}^{-1}(u)\right)}=\frac{(1-u)^{1 / n-1}}{n f\left(F^{-1}\left(1-(1-u)^{1 / n}\right)\right)}, \\
& \frac{1}{g_{1: n}\left(G_{1: n}^{-1}(u)\right)}=\frac{(1-u)^{1 / n-1}}{n g\left(G^{-1}\left(1-(1-u)^{1 / n}\right)\right)} .
\end{aligned}
$$

Now, we can write $X_{1: n} \leq_{\text {imit }} Y_{1: n}$ if, and only if,

$$
\begin{aligned}
& \int_{0}^{p^{\prime}} \frac{q^{\prime} d F_{1: n}^{-1}\left(q^{\prime}\right)}{g_{1: n}\left(G_{1: n}^{-1}\left(p^{\prime}\right)\right)} \geq \int_{0}^{p^{\prime}} \frac{q^{\prime} d G_{1: n}^{-1}\left(q^{\prime}\right)}{f_{1: n}\left(F_{1: n}^{-1}\left(p^{\prime}\right)\right)}, \\
& \forall p^{\prime} \in(0,1),
\end{aligned}
$$

or, equivalently, if

$$
\begin{aligned}
& \int_{0}^{p^{\prime}} \frac{q^{\prime} d F^{-1}\left(1-\left(1-q^{\prime}\right)^{1 / n}\right)}{n g\left(G^{-1}\left(1-\left(1-p^{\prime}\right)^{1 / n}\right)\right)} \\
& \quad \geq \int_{0}^{p^{\prime}} \frac{q^{\prime} d G^{-1}\left(1-\left(1-q^{\prime}\right)^{1 / n}\right)}{n f\left(F^{-1}\left(1-\left(1-p^{\prime}\right)^{1 / n}\right)\right)}, \quad \forall p^{\prime} \in(0,1) .
\end{aligned}
$$

By making the change of variable $q=1-\left(1-q^{\prime}\right)^{1 / n}$ and also taking $q=1-\left(1-q^{\prime}\right)^{1 / n}$ in (49), we conclude that $X_{1: n} \leq_{\text {imit }} Y_{1: n}$ holds if, and only if, $X_{1: n} \leq_{\text {imit }} Y_{1: n}$, if, and only if,

$$
\int_{0}^{p} \frac{\psi(q) d F^{-1}(q)}{g\left(G^{-1}(p)\right)} \geq \int_{0}^{p} \frac{\psi(q) d G^{-1}(q)}{f\left(F^{-1}(p)\right)},
$$

where

$$
\psi(q)=(1-q)^{n-1}\left(1-(1-q)^{n}\right), \quad q \in(0,1) .
$$

From (50), we know that $X_{1: n} \leq_{\text {imit }} Y_{1: n}$ if, and only if,

$$
\int_{0}^{1} \frac{\psi(q)}{q} d W(q, p) \geq 0, \quad \forall p \in(0,1),
$$

in which $W(q, p)$ is defined as before in Theorem 9. On the other side, we obtain by assumption, as in the proof of Theorem 9, that $\int_{0}^{q^{*}} d W(q, p) \geq 0$, for all $p, q^{*} \in(0,1)$. Since

$$
\frac{\psi(q)}{q}=(1-q)^{n-1}\left(1+(1-q)+\cdots+(1-q)^{n-1}\right),
$$

is nonnegative and decreasing for any $q \in(0,1)$, thus an application of Lemma 2 (ii) leads to (52). Hence, the proof is completed.

Theorem 13. In the setting of Theorem 12,

$$
\begin{gathered}
X_{n: n} \leq_{i m i t} Y_{n: n} \Longrightarrow \\
X \leq_{i m i t} Y .
\end{gathered}
$$

Proof. Suppose that $F_{n: n}$ and $G_{n: n}$ denote the distribution functions of $X_{n: n}$ and $Y_{n: n}$, respectively, which are given by

$$
\begin{aligned}
& F_{n: n}(t)=F^{n}(x), \\
& G_{n: n}(t)=G^{n}(t),
\end{aligned}
$$

$$
t \geq 0,
$$

leading to

$$
\begin{aligned}
F_{n: n}^{-1}(u) & =F^{-1}\left(u^{1 / n}\right), \\
G_{n: n}^{-1}(u) & =G^{-1}\left(u^{1 / n}\right)
\end{aligned}
$$

for any $u \in(0,1)$.

By considering $f_{n: n}$ and $g_{n: n}$ as the density functions of $X_{n: n}$ and $Y_{n: n}$, respectively, for any $u \in(0,1)$, the following holds:

$$
\begin{aligned}
& \frac{1}{f_{n: n}\left(F_{n: n}^{-1}(u)\right)}=\frac{u^{1 / n-1}}{n f\left(F^{-1}\left(u^{1 / n}\right)\right)}, \\
& \frac{1}{g_{n: n}\left(G_{n: n}^{-1}(u)\right)}=\frac{u^{1 / n-1}}{n g\left(G^{-1}\left(u^{1 / n}\right)\right)} .
\end{aligned}
$$

Now, let us denote $d W^{*}(q, p)=I[q<p] w^{*}(q, p)$, for any $p, q \in(0,1)$, such that

$$
w^{*}(q, p)=q^{n}\left(\frac{d F^{-1}(q)}{g\left(G^{-1}(p)\right)}-\frac{d G^{-1}(q)}{f\left(F^{-1}(p)\right)}\right) .
$$

Using the identities (56) and (57), it follows, by similar arguments to those provided for the proof of Theorem 12, that

$$
\int_{0}^{p} \frac{q^{n} d F^{-1}(q)}{g\left(G^{-1}(p)\right)} \geq \int_{0}^{p} \frac{q^{n} d G^{-1}(q)}{f\left(F^{-1}(p)\right)}, \quad \forall p \in(0,1),
$$

which is equivalent to

$$
\int_{0}^{p} d W^{*}(q, p) \geq 0, \quad \forall p \in(0,1) .
$$

Thus,

$$
\int_{0}^{q^{*}} d W^{*}(q, p) \geq 0, \quad \text { for any } q^{*} \geq p \in(0,1) .
$$

In addition, if $X_{n: n} \leq_{\text {imit }} Y_{n: n}$ then $\int_{0}^{p} q^{n} d G^{-1}(q) / \int_{0}^{p} q^{n} d F^{-1}(q)$ is increasing in $p \in(0,1)$. In view of (59), for all $q^{*} \leq p \in$ $(0,1)$,

$$
\frac{\int_{0}^{q^{*}} q^{n} d G^{-1}(q)}{\int_{0}^{q^{*}} q^{n} d F^{-1}(q)} \leq \frac{\int_{0}^{p} q^{n} d G^{-1}(q)}{\int_{0}^{p} q^{n} d F^{-1}(q)} \leq \frac{f\left(F^{-1}(p)\right)}{g\left(G^{-1}(p)\right)},
$$

which means that

$$
\int_{0}^{q^{*}} d W^{*}(q, p) \geq 0, \quad \text { for any } q^{*} \leq p \in(0,1) .
$$


Combining (61) and (63), we have

$$
\int_{0}^{q^{*}} d W^{*}(q, p) \geq 0, \quad \forall p, q^{*} \in(0,1) .
$$

By Lemma 2 (ii), it is implied that

$$
\int_{0}^{1}\left(\frac{1}{q}\right)^{n-1} d W^{*}(q, p) \geq 0, \quad \forall p \in(0,1),
$$

which is equivalent to saying that $X \leq_{\text {imit }} Y$.

2.4. Comparisons of Mixtures of a Family of Semiparametric Distributions. In this subsection, preservation of the order " $\leq_{\text {imit }}$ " under mixtures of a typical family of semiparametric distributions which includes some well-known models in reliability and survival analysis is established and vice versa. Some examples of interest are given to authenticate the results. Semiparametric distributions that are distinguished by having a parameter that is itself a distribution function and thereby extending the family from which this distribution came play an important role in statistical literature (cf. Powell [25] and Marshall and Olkin [6]). In this work, we consider a typical family of semiparametric distributions that includes some well-known models such as proportional hazards and proportional reversed hazards families. Suppose that $X$ is a random variable with distribution function $F$, and let $\theta$ be a parameter with parameter space $\chi$, where $\chi$ is an arbitrary subset of $R$ (countable or uncountable). We focus on a general semiparametric family with the underlying distribution $F$ that provides a way to add a new parameter $\theta$ through the relation

$$
F(x \mid \theta)=\gamma(F(x), \theta), \quad x \in \mathbb{R}, \theta \in \chi,
$$

where

$$
\begin{aligned}
\gamma:[0,1] & \longrightarrow[0,1], \\
d & \longrightarrow \gamma(d, \theta),
\end{aligned}
$$

being a nonnegative one to one function satisfying the following conditions:

(i) $0 \leq \gamma(d, \theta) \leq 1$, for all $d \in[0,1]$ and $\theta \in \chi$.

(ii) $\gamma(0, \theta)=0$, for all $\theta \in \chi$.

(iii) $\gamma(1, \theta)=1$, for all $\theta \in \chi$.

(iv) $\gamma$ is strictly increasing and right continuous for all $\theta \in$ $\chi$.

Under conditions (i)-(iv), $F(\cdot \mid \theta)$ in (66) is a distribution function for every $\theta \in \chi$. By choosing a function

$$
\begin{aligned}
\phi:[0,1] & \longrightarrow[0, \infty), \\
t & \longrightarrow \phi(t, \theta),
\end{aligned}
$$

one obtains a general form for the function $\gamma$ in (66) as

$$
\gamma(d, \theta)=\frac{\int_{0}^{d} \phi(t, \theta) d t}{\int_{0}^{1} \phi(t, \theta) d t} .
$$

Below we provide some choices for the function $\phi$ in (69) leading to several important models. (i) Order statistics: $\phi(t, \theta)=t^{i-1}(1-t)^{\theta-i}, i=1,2, \ldots, \theta$ with $\theta \in N$.

(ii) Lower records: $\phi(t, \theta)=(-\ln (t))^{\theta-1}$, with $\theta \in N$.

(iii) Upper records: $\phi(t, \theta)=(-\ln (1-t))^{\theta-1}$, with $\theta \in N$.

(iv) Proportional hazards: $\phi(t, \theta)=1-(1-t)^{\theta}$, where $\theta \in R^{+}$.

(v) Proportional reversed hazards: $\phi(t, \theta)=t^{\theta}$, where $\theta \epsilon$ $R^{+}$.

(vi) Upper tail distribution: $\phi(t, \theta)=I(t \geq \theta)$ with $\theta \epsilon$ $(0,1)$.

(vii) Lower tail distribution: $\phi(t, p)=I(t<\theta)$ with $\theta \in$ $(0,1)$.

In many practical situations the parameter $\theta$ may not be constant due to various reasons, and the contingency of heterogeneity is sometimes unpredictable and unexplained. The heterogeneity may often not be possible to be neglected. Further, it mostly happens that data from several populations is mixed and information about which subpopulation gave rise to individual data points is unavailable. There are numerous cases in practical situations in which data are coming from various sources and the statistician, therefore, needs to be aware of the initial source from which data have been derived. The mixture of the families of distributions according to a proper mixing rule is useful to model such data sets in frail populations. In the continuing part of the paper, the mixture of the family of semiparametric distributions in (66) is considered. Formally, let $\Theta$ be a random variable (discrete or continuous) with support in $\chi$ having distribution function $\Lambda$. Let $X$ and $Y$ be two nonnegative random variables with distributions $F$ and $G$, respectively. Then, we shall denote by $X^{*}$ and $Y^{*}$ two random variable with distributions

$$
\begin{aligned}
& F^{*}(x)=\int_{\chi} \gamma(F(x), \theta) d \Lambda(\theta), \quad x \in \mathbb{R}, \\
& G^{*}(x)=\int_{\chi} \gamma(G(x), \theta) d \Lambda(\theta), \quad x \in \mathbb{R},
\end{aligned}
$$

respectively. Before stating the main result of this subsection, we introduce some notations. For a given function $\gamma$ satisfying the conditions (i)-(iv) as before, set

$$
L(d)=\int_{\chi} \gamma(d, \theta) d \Lambda(\theta), \quad d \in[0,1]
$$

which is nonnegative, strictly increasing, and right continuous. Note that we could write $L(d)=E[\gamma(d, \Theta)]$ for $d \in[0,1]$. In view of (70) and (71), we have

$$
\begin{aligned}
& F^{*}(x)=L(F(x)), \\
& G^{*}(x)=L(G(x)) .
\end{aligned}
$$

The following result, under some appropriate assumptions, translates the imit order in $X$ and $Y$ to the imit order between $X^{*}$ and $Y^{*}$ and vice versa. 
Theorem 14. Let $X$ and $Y$ be two nonnegative random variables with absolutely continuous distribution functions $F$ and $G$, and let $X^{*}$ and $Y^{*}$ have distributions $F^{*}$ and $G^{*}$ as in (70) and (71), respectively.

(i) If $L(d) / d$ is decreasing in $d \in(0,1]$ then

$$
\begin{gathered}
X \leq_{i m i t} Y \Longrightarrow \\
X^{*} \leq_{i m i t} Y^{*} .
\end{gathered}
$$

(ii) If $L(d) / d$ is increasing in $d \in(0,1]$ then

$$
\begin{gathered}
X^{*} \leq_{i m i t} Y^{*} \Longrightarrow \\
X \leq_{i m i t} Y .
\end{gathered}
$$

Proof. First denote by $f^{*}$ and $g^{*}$ the density functions of $X^{*}$ and $Y^{*}$, respectively, and denote by $L^{-1}$ the right continuous inverse function of $L$ in (72) which is given by $L^{-1}(q)=$ $\inf \{x \in(0,1) \mid L(x) \geq q\}$. Appealing to the identities in (73), for any $u \in(0,1)$, we have

$$
\begin{aligned}
F^{*-1}(u) & =F^{-1}\left(L^{-1}(u)\right), \\
G^{*-1}(u) & =G^{-1}\left(L^{-1}(u)\right), \\
\frac{1}{f^{*}\left(F^{*-1}(u)\right)} & =\frac{1}{L^{\prime}\left(L^{-1}(u)\right) f\left(F^{-1}\left(L^{-1}(u)\right)\right)},
\end{aligned}
$$

and, similarly,

$$
\frac{1}{g^{*}\left(G^{*-1}(u)\right)}=\frac{1}{L^{\prime}\left(L^{-1}(u)\right) g\left(G^{-1}\left(L^{-1}(u)\right)\right)} .
$$

Therefore, $X^{*} \leq_{\text {imit }} Y^{*}$ if, and only if,

$$
\begin{aligned}
& \int_{0}^{p} \frac{q d F^{-1}\left(L^{-1}(q)\right)}{L^{\prime}\left(L^{-1}(p)\right) g\left(G^{-1}\left(L^{-1}(p)\right)\right)} \\
& \quad \geq \int_{0}^{p} \frac{q d G^{-1}\left(L^{-1}(q)\right)}{L^{\prime}\left(L^{-1}(p)\right) f\left(F^{-1}\left(L^{-1}(p)\right)\right)},
\end{aligned}
$$

$$
\forall p \in(0,1),
$$

or, equivalently, if

$$
\begin{array}{r}
\int_{0}^{p} \frac{q d F^{-1}\left(L^{-1}(q)\right)}{g\left(G^{-1}\left(L^{-1}(p)\right)\right)} \geq \int_{0}^{p} \frac{q d G^{-1}\left(L^{-1}(q)\right)}{f\left(F^{-1}\left(L^{-1}(p)\right)\right)}, \\
\forall p \in(0,1) .
\end{array}
$$

Since $L^{-1}(0)=0$ thus by making a proper change of variable, one observes from (79) that $X^{*} \leq_{\mathrm{imit}} Y^{*}$ is equivalent to

$$
\int_{0}^{p} \frac{L(q) d F^{-1}(q)}{g\left(G^{-1}(p)\right)} \geq \int_{0}^{p} \frac{L(q) d G^{-1}(q)}{f\left(F^{-1}(p)\right)}
$$

which holds if, and only if,

$$
\int_{0}^{1} \frac{L(q)}{q} d W(q, p) \geq 0, \quad \forall p \in(0,1),
$$

in which $W(q, p)$ is defined as in the proof of Theorem 9 , for which (32) holds provided that $X \leq_{\text {imit }} Y$. By the assumption that $L(q) / q$ is decreasing we can use Lemma 2 (ii) to conclude (81). This ends the proof of (i). Now, assume that $X^{*} \leq_{\text {imit }} Y^{*}$ and denote $d W^{* *}(q, p)=I[q<p] w^{* *}(q, p)$ with

$$
w^{* *}(q, p)=L(q)\left(\frac{d F^{-1}(q)}{g\left(G^{-1}(p)\right)}-\frac{d G^{-1}(q)}{f\left(F^{-1}(p)\right)}\right) .
$$

From (80), we see that

$$
\int_{0}^{q^{*}} d W^{* *}(q, p) \geq 0, \quad \forall q^{*}>p \in(0,1) .
$$

Besides, if $X^{*} \leq_{\text {imit }} Y^{*}$ then

$$
\frac{\int_{0}^{p} L(q) d G^{-1}(q)}{\int_{0}^{p} L(q) d F^{-1}(q)} \text { is increasing in } p \in(0,1) .
$$

It thus follows using (80) that

$$
\begin{aligned}
\frac{\int_{0}^{q^{*}} L(q) d G^{-1}(q)}{\int_{0}^{q^{*}} L(q) d F^{-1}(q)} & \leq \frac{\int_{0}^{p} L(q) d G^{-1}(q)}{\int_{0}^{p} L(q) d F^{-1}(q)} \\
& \leq \frac{f\left(F^{-1}(p)\right)}{g\left(G^{-1}(p)\right)},
\end{aligned}
$$

$$
\forall q^{*} \leq p \in(0,1),
$$

from which we get

$$
\int_{0}^{q^{*}} d W^{* *}(q, p) \geq 0, \quad \forall q^{*} \leq p \in(0,1) .
$$

Therefore, we conclude from (83) and (86) that

$$
\int_{0}^{q^{*}} d W^{* *}(q, p) \geq 0, \quad \forall p, q^{*} \in(0,1) .
$$

From assumption $q / L(q)$ is decreasing in $q \in(0,1)$. Now, using Lemma 2 (ii), it is deduced that

$$
\int_{0}^{1} \frac{q}{L(q)} d W^{* *}(q, p) \geq 0, \quad \forall p \in(0,1) .
$$

That is, $X \leq_{\text {imit }} Y$. The proof of (ii) is complete.

Example 15. Let $X_{1}, X_{2}, \ldots$ and $Y_{1}, Y_{2}, \ldots$ each be a sequence of independent and identically distributed random variables with distribution functions $F$ and $G$, respectively. Let $N$ be a positive integer-valued random variable, independent of $X_{i}$ 's and of $Y_{i}$ 's with probability mass function $\lambda(n)=P(N=n)$, $n \in N$ in which $N$ is the set of natural numbers. Denote by

$$
\begin{aligned}
& X_{1: N}=\min \left\{X_{1}, X_{2}, \ldots, X_{N}\right\}, \\
& Y_{1: N}=\min \left\{Y_{1}, Y_{2}, \ldots, Y_{N}\right\}
\end{aligned}
$$


the extreme minimum order statistics of the two sequences and denote their distribution functions by $F_{1: N}$ and $G_{1: N}$, respectively. It can be easily shown that

$$
\begin{aligned}
& F_{1: N}(x)=E[\gamma(F(x), N)], \\
& G_{1: N}(x)=E[\gamma(G(x), N)],
\end{aligned}
$$

where

$$
\gamma(d, n)=1-(1-d)^{n}, \quad d \in(0,1) .
$$

Now, since for any $d \in(0,1)$ and $n \in N$, we have

$$
\frac{\gamma(d, n)}{d}=1+(1-d)+\cdots+(1-d)^{n-1},
$$

which is decreasing in $d \in(0,1)$, for all $n \in N$, thus $L(d) / d$ will also be decreasing in $d \in(0,1)$ where $L(d)=E[\gamma(d, N)]$ as in (72). Now, Theorem 14 (i) is applicable and provides

$$
\begin{gathered}
X_{1} \leq_{\text {imit }} Y_{1} \Longrightarrow \\
X_{1: N} \leq_{\text {imit }} Y_{1: N} .
\end{gathered}
$$

In a similar manner, if we denote by

$$
\begin{aligned}
& X_{N: N}=\max \left\{X_{1}, X_{2}, \ldots, X_{N}\right\}, \\
& Y_{N: N}=\max \left\{Y_{1}, Y_{2}, \ldots, Y_{N}\right\}
\end{aligned}
$$

the extreme maximum order statistics of the two sequences then they have respective distribution functions

$$
\begin{aligned}
& F_{N: N}(x)=E[\gamma(F(x), N)], \\
& G_{N: N}(x)=E[\gamma(G(x), N)],
\end{aligned}
$$

where

$$
\gamma(d, n)=d^{n}, \quad d \in(0,1)
$$

Because $\gamma(d, n) / d=d^{n-1}$ is increasing in $d \in(0,1)$, for all $n \in N, L(d) / d$ will be increasing in $d \in(0,1)$. Hence, by Theorem 14 (ii),

$$
\begin{gathered}
X_{N: N} \leq_{\text {imit }} Y_{N: N} \Longrightarrow \\
X_{1} \leq_{\text {imit }} Y_{1} .
\end{gathered}
$$

Example 16. Marshall and Olkin [26], as a general method of introducing a parameter (tilt parameter) to give more flexibility in modelling, discussed new semiparametric families of distributions. Given a distribution function $F$, they supposed that

$$
F(x \mid \theta)=\frac{F(x)}{1-(1-\theta)(1-F(x))}, \quad-\infty<x<+\infty,
$$

where $\theta$ is a positive parameter. By making a comparison between (66) and (98) we see that $\gamma$ must be chosen as

$$
\gamma(d, \theta)=\frac{d}{1-(1-\theta)(1-d)} .
$$

Recently, Nanda and Das [27] considered a mixture form of the distribution (98) where $\theta$ is taken as a random variable in order to investigate closure properties of the model with respect to some stochastic orders. For two given random variables $X$ and $Y$ with respective distribution functions $F$ and $G$, we assume that $X^{*}$ and $Y^{*}$ are random variables with distributions

$$
\begin{aligned}
& F^{*}(x)=E[\gamma(F(x), \Theta)], \\
& G^{*}(x)=E[\gamma(G(x), \Theta)],
\end{aligned}
$$

respectively, where $\Theta$ is a nonnegative random variable. It is possible to observe $L(d) / d$, when $\chi \subseteq[0,1]$, is decreasing in $d \in(0,1)$, and in parallel, when $\chi \subseteq[1, \infty), L(d) / d$ is increasing in $d \in(0,1)$. Therefore, by Theorem 14 (i), if $P(\Theta \leq$ $1)=1$, then $X \leq_{\text {imit }} Y$ implies $X^{*} \leq_{\text {imit }} Y^{*}$ and on the other hand when $P(\Theta \geq 1)=1$, using Theorem 14 (ii), $X^{*} \leq_{\text {imit }} Y^{*}$ gives $X \leq_{\text {imit }} Y$.

\section{Conflicts of Interest}

All of the authors declare that there are no conflicts of interest related to this paper.

\section{Acknowledgments}

The first and the third authors would like to extend their sincere appreciation to the Deanship of Scientific Research at King Saud University for funding this research, Group no. RGP-1435-036.

\section{References}

[1] M. Shaked and J. G. Shanthikumar, Stochastic Orders, Springer Series in Statistics, Springer, New York, NY, USA, 2007.

[2] A. Müller and D. Stoyan, Comparison Methods for Stochastic Models and Risks, John Wiley, New York, NY, USA, 2002.

[3] F. Belzunce, C. Martínez-Riquelme, and J. Mulero, An Introduction to Stochastic Orders, Academic Press, 2015.

[4] N. U. Nair, P. G. Sankaran, and N. Balakrishnan, Quantile-Based Reliability Analysis, Birkhuser, Basel, Switzerland, 2013.

[5] W. R. Van Zwet, Convex Transformations of Random Variables, vol. 7, Mathematisch centrum, 1964.

[6] A. W. Marshall and I. Olkin, Life Distributions, Springer, New York, NY, USA, 2007.

[7] S. Kochar and M. Xu, "Comparisons of parallel systems according to the convex transform order," Journal of Applied Probability, vol. 46, no. 2, pp. 342-352, 2009.

[8] G. Barmalzan and A. T. Payandeh Najafabadi, "On the convex transform and right-spread orders of smallest claim amounts," Insurance: Mathematics \& Economics, vol. 64, pp. 380-384, 2015.

[9] S. C. Kochar and D. P. Wiens, "Partial orderings of life distributions with respect to their aging properties," Naval Research Logistics (NRL), vol. 34, no. 6, pp. 823-829, 1987.

[10] C.-D. Lai and M. Xie, "Stochastic ageing and dependence for reliability," Stochastic Ageing and Dependence for Reliability, pp. 1-418, 2006.

[11] S. C. Kochar, "On extensions of DMRL and related partial orderings of life distributions," Communications in Statistics. Stochastic Models, vol. 5, no. 2, pp. 235-245, 1989. 
[12] D. T. Kang and L. Yan, "Further results on the DMRL ordering," Chinese Journal of Applied Probability and Statistics, vol. 32, no. 1, pp. 69-88, 2016.

[13] J. Bartoszewicz, "Mixtures of exponential distributions and stochastic orders," Statistics \& Probability Letters, vol. 57, no. 1, pp. 23-31, 2002.

[14] P. J. Boland, T. Hu, M. Shaked, and J. G. Shanthikumar, "Stochastic ordering of order statistics II," in Modeling Uncertainty, pp. 607-623, Springer US, 2005.

[15] J. Bartoszewicz and M. Skolimowska, "Preservation of classes of life distributions and stochastic orders under weighting," Statistics \& Probability Letters, vol. 76, no. 6, pp. 587-596, 2006.

[16] J. Bartoszewicz and M. Skolimowska, "Preservation of stochastic orders under mixtures of exponential distributions," Probability in the Engineering and Informational Sciences, vol. 20, no. 4, pp. 655-666, 2006.

[17] S. Kochar and M. Xu, "On the skewness of order statistics with applications," Annals of Operations Research, vol. 212, pp. 127138, 2014.

[18] F. Belzunce, J. F. Pinar, J. M. Ruiz, and M. A. Sordo, "Comparison of risks based on the expected proportional shortfall," Insurance: Mathematics \& Economics, vol. 51, no. 2, pp. 292-302, 2012.

[19] A. Arriaza, M. A. Sordo, and A. Súarez-Llorens, "Comparing residual lives and inactivity times by transform stochastic orders," IEEE Transactions on Reliability, vol. 66, no. 2, pp. 366372, 2017.

[20] M. Kayid and I. A. Ahmad, "On the mean inactivity time ordering with reliability applications," Probability in the Engineering and Informational Sciences, vol. 18, no. 3, pp. 395-409, 2004.

[21] I. Jewitt, "Choosing between risky prospects: the characterization of comparative statics results, and location independent risk," Management Science, vol. 35, no. 1, pp. 60-70, 1989.

[22] S. Karlin, Total Positivity, vol. 1, Stanford University Press, Stanford, Calif, USA, 1968.

[23] F. Belzunce, C. Martínez-Riquelme, J. M. Ruiz, and M. A. Sordo, "On the comparison of relative spacings with applications," Methodology and Computing in Applied Probability, vol. 19, no. 2, pp. 357-376, 2017.

[24] F. Belzunce and C. Martínez-Riquelme, "On sufficient conditions for the comparison of some quantile-based measures," Communications in Statistics-Theory and Methods, vol. 46, no. 13, pp. 6512-6527, 2017.

[25] J. L. Powell, "Chapter 41 Estimation of semiparametric models," Handbook of Econometrics, vol. 4, pp. 2443-2521, 1994.

[26] A. W. Marshall and I. Olkin, "A new method for adding a parameter to a family of distributions with application to the exponential and Weibull families," Biometrika, vol. 84, no. 3, pp. 641-652, 1997.

[27] A. K. Nanda and S. Das, "Stochastic orders of the MarshallOlkin extended distribution," Statistics \& Probability Letters, vol. 82, no. 2, pp. 295-302, 2012. 


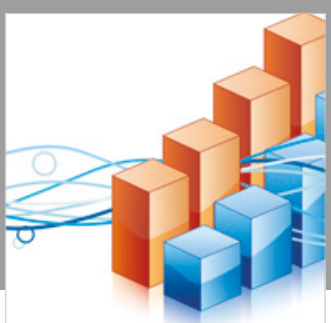

Advances in

Operations Research

\section{-n-m}
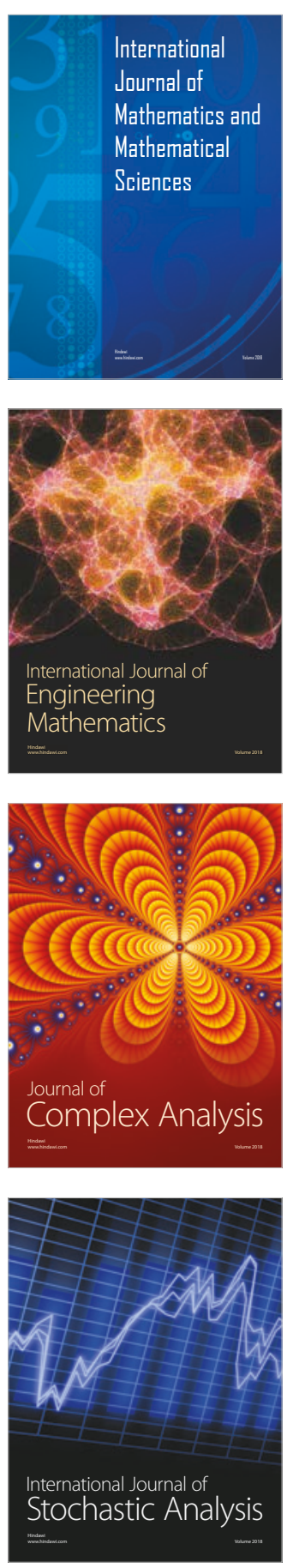
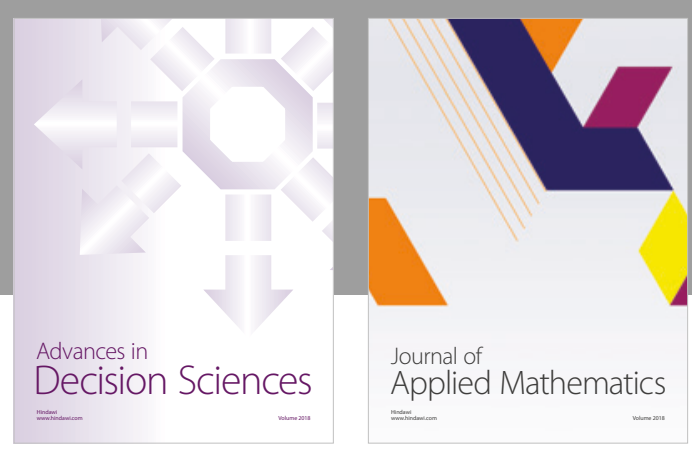

Journal of

Applied Mathematics
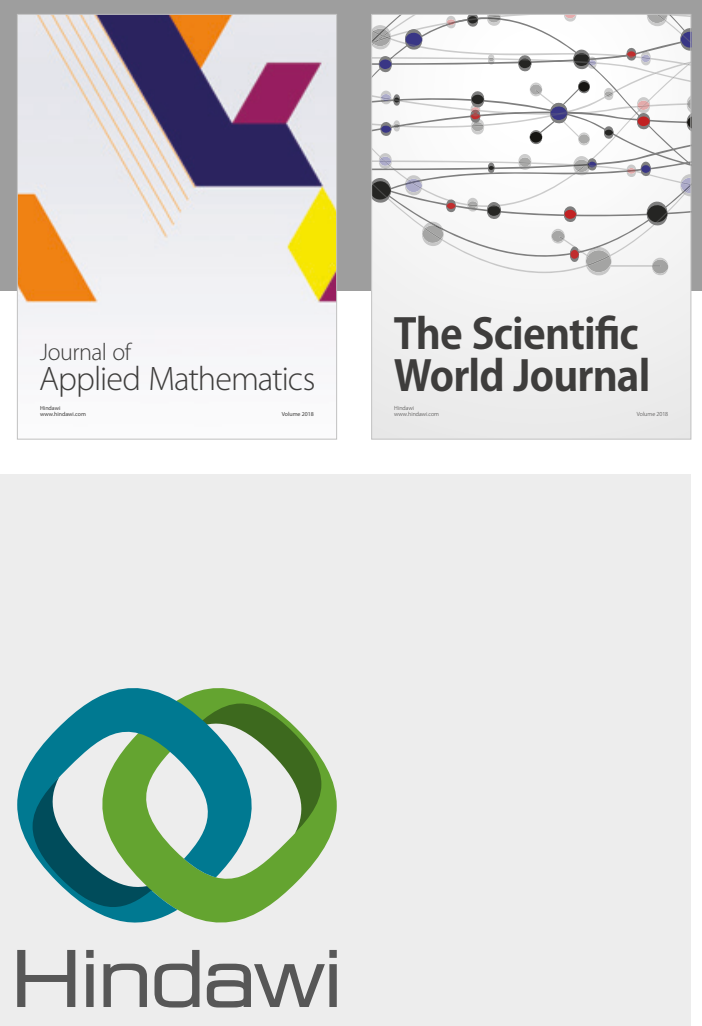

Submit your manuscripts at

www.hindawi.com

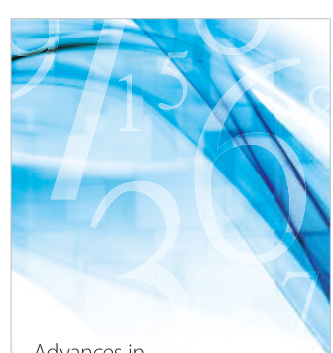

Advances in
Numerical Analysis
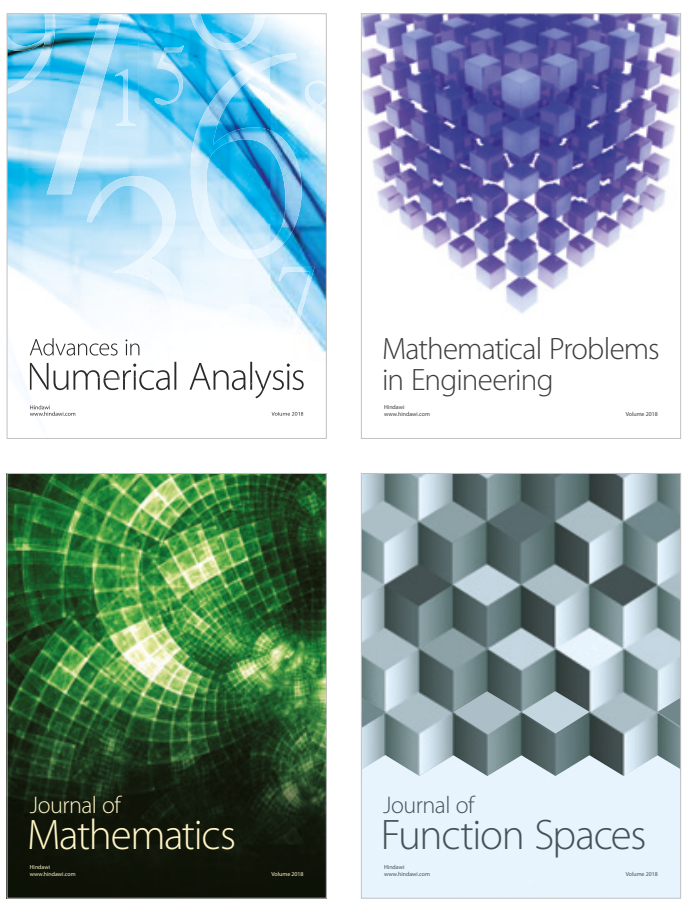

Mathematical Problems in Engineering

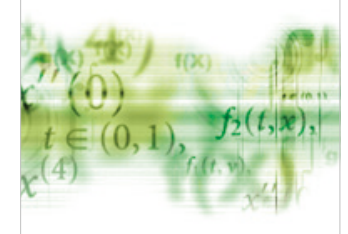

International Journal of

Differential Equations

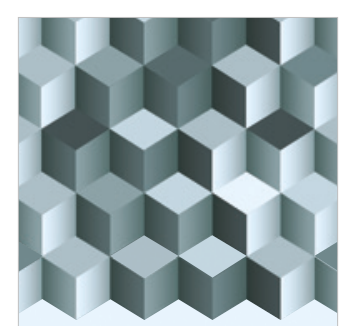

Journal of

Function Spaces

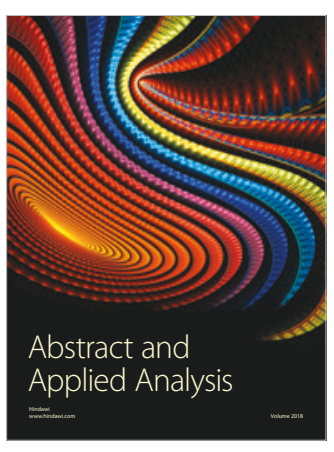

The Scientific

World Journal

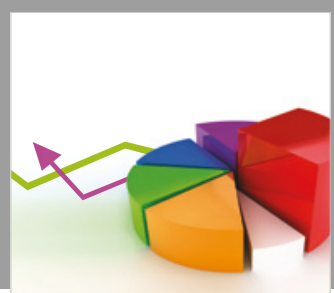

Journal of

Probability and Statistics
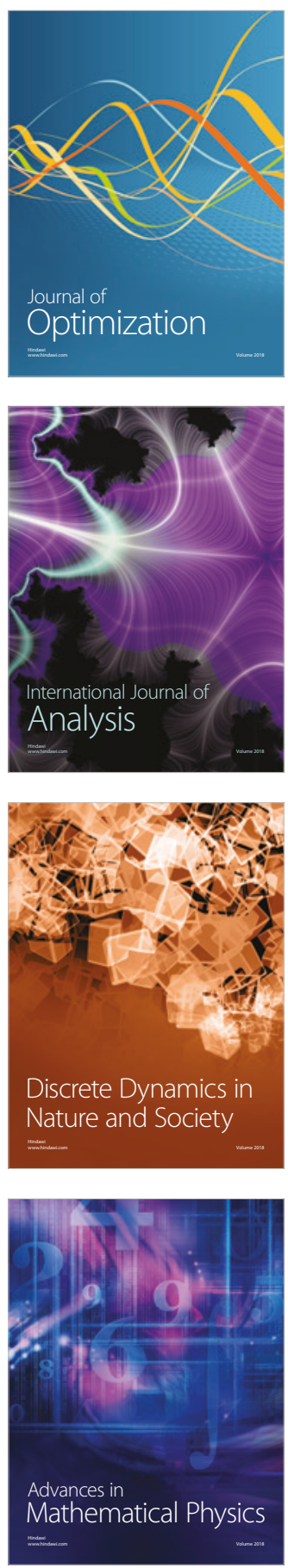